\title{
FAM98A promotes proliferation of non-small cell lung cancer cells via the P38-ATF2 signaling pathway
}

This article was published in the following Dove Press journal:

Cancer Management and Research

\author{
Rui Zheng' \\ Quanbo Liu' \\ Tianxu Wang' \\ Lili Wang' \\ Yong Zhang ${ }^{2}$ \\ 'Department of Respiratory \\ Medicine, Shengjing Hospital of China \\ Medical University, Shenyang, China; \\ ${ }^{2}$ Department of Pathology, Cancer \\ Hospital of China Medical University, \\ Liaoning Cancer Hospital \& Institute, \\ Shenyang, China
}

Correspondence: Rui Zheng Department of Respiratory Medicine, Shengjing Hospital of China Medical University, No. 36 Sanhao Street, Heping District, Shenyang II 0004, China Email zhengr@sj-hospital.org

Yong Zhang

Department of Pathology, Cancer Hospital of China Medical University, Liaoning Cancer Hospital \& Institute, No. 44 Xiaoheyan Road, Dadong District, Shenyang II 0042, China

Email zhangyong@cancerhosp-In-cmu.com
Background: FAM98A, a novel protein, is expressed in ovarian and colorectal cancer tissues. However, the association between FAM98A expression and the clinicopathological characteristics of non-small cell lung cancer (NSCLC) remains undetermined.

Materials and methods: The FAM98A expression pattern was determined in NSCLC samples and corresponding adjacent normal lung tissues using immunohistochemical staining and Western blotting. The association of FAM98A expression with clinicopathological characteristics was measured in 131 NSCLC samples. Finally, the overexpression and inhibition of FAM98A was performed in the A549 and SPC-A1 cell lines to explore its role in the development of lung cancer. Results: Western blot analysis of 20 paired NSCLC samples showed that expression of FAM98A was higher in lung cancer tissues than in the corresponding adjacent normal lung tissues $(p<0.05)$. Immunohistochemical staining of 128 NSCLC specimens showed that expression of FAM98A was significantly higher in lung cancer samples than in adjacent normal lung tissues $(118 / 128$ vs $10 / 128 ; p<0.001)$. Positive expression of FAM98A was significantly related to tumor TNM stage $(p<0.05)$ and lymph node metastasis $(p<0.001)$. Additionally, overexpression of FAM98A induced an increase in the expression of phosphorylated P38, phosphorylated ATF2, and cyclin D1, which promoted proliferation of lung cancer cells. Correspondingly, the effects of FAM98A overexpression were reversed by administration of a specific inhibitor of phosphorylated P38. Conclusion: FAM98A was overexpressed in the cytoplasm of NSCLC samples and correlated with advanced TNM staging and lymph node metastasis. Thus, FAM98A increases the expression of cyclin D1 by activating the P38-ATF2 signaling pathway and subsequently enhancing tumor cell proliferation; these results are promising and need further validation.

Keywords: ATF2, TNM stage, lymph node metastasis

\section{Introduction}

The incidence and mortality rate of lung cancer are among the highest of all tumors worldwide. ${ }^{1}$ Nearly $70 \%$ of patients diagnosed with lung cancer are in a late stage, with local diffusion and/or distant transfer. ${ }^{2}$ Non-small cell lung cancer (NSCLC) accounts for almost $80 \%$ of lung cancer diagnoses. Therefore, there is a need to explore the factors underlying the pathogenesis of lung cancer to improve clinical treatment strategies.

FAM98A, a novel protein, was reported to be a member of the recently identified $\mathrm{NN}-\mathrm{CH}$ family and is related to the yeast outer kinetochore components NDC80 and NUF2. ${ }^{3}$ The NN-CH family is defined by possession of the divergent calponin homology domain, which contains a different $\mathrm{N}$-terminal domain but a similar $\mathrm{CH}$ 
domain. Additionally, it contains 7 repeated amino acid sequences, which are predicted to form a circular arrangement. This family includes NDC80, NUF2, FAM98A-C, CCDC22, CCDC93, C14orf166, NDC80/HEC1, NUF2, IFT81, IFT57, and CLUAP1. ${ }^{3}$ C14orf166, a member of the $\mathrm{NN}-\mathrm{CH}$ family, is upregulated in bladder cancer cells and tissues, and could promote the proliferation of bladder tumor cells by regulating the cell cycle. ${ }^{4}$ FAM98A forms a molecular complex with PLHKEM1, DEF8, and NDEL1 that regulates lysosome positioning and secretion through RAB7. ${ }^{5}$ Moreover, FAM98A is arginine-methylated by protein arginine methyltransferase 1 (PRMT1) and is essential for malignancy of ovarian cancer cells. ${ }^{6}$ The kinetochore is composed of many conserved protein complexes that guide its specification, assembly, attachment to spindle microtubules, and regulation of chromosome segregation. ${ }^{7}$ Furthermore, the kinetochore provides an essential site of attachment for spindle microtubules during mitosis. Additionally, it controls a cell cycle checkpoint. ${ }^{8}$ As a microtubule-binding protein localized to centromeres, NDC80 acts on the checkpoint and is responsible for connection to non-stationary microtubules by polymerization and disaggregation. ${ }^{9,10}$

Therefore, we speculated that FAM98A is related to connection of centromeres and microtubules and potentially influences cell proliferation. We explored the role of FAM98A in the etiology of NSCLC by examining its expression and subcellular location in lung cancer tissues and analyzed the corresponding clinicopathologic factors using immunohistochemistry, Western blotting, MTT assay, colony formation assay, and immunofluorescent staining.

\section{Materials and methods}

\section{Tissue samples}

Written informed consent was obtained from all participants. The study was approved by the Ethics Committee of China Medical University. A total of 131 tissue samples were collected (102 males and 29 females, median age=60 years). The patients underwent complete surgical excision of the tumor at the First Hospital of China Medical University from 2008 to 2009 after being diagnosed with NSCLC. Corresponding noncancerous lung tissues were available for 128 of the patients. The patients did not undergo neoadjuvant radiotherapy or chemotherapy prior to surgery. Histological diagnosis and grading were performed according to the World Health Organization guidelines for classification of lung tumors published in 2015. ${ }^{11}$ All 131 specimens were evaluated for histological subtype, differentiation, and tumor stage. Tumor staging was performed according to the seventh edition of the International Union against Cancer TNM Staging System for Lung Cancer. ${ }^{12}$ Of the 131 patients, 63 were $>60$ years old and $68<60$ years of age. Of the 131 tissue samples, 60 were diagnosed as squamous cell lung carcinoma, 62 as lung adenocarcinoma, and 9 cases as other histological types of lung cancer. There were 15 welldifferentiated, 59 moderately differentiated, and 57 poorly differentiated tumors. Lymph node metastases were found in 58 cases. A total of 90 cases were classified as TNM stages I-II and 41 as stage III.

Additionally, from the 131 tissue samples, 20 fresh tissue specimens were selected that included both tumor and corresponding adjacent noncancerous tissue. The specimens were stored at $-80^{\circ} \mathrm{C}$ immediately after resection and then used for the following protein extraction.

\section{Cell culture}

The human bronchial epithelial (HBE) cell line was purchased from the American Type Culture Collection (Manassas, VA, USA). The A549, H1299, H460, 973, 83A, LK2, and SPC-A1 cell lines were obtained from the Shanghai Cell Bank (Shanghai, China). All cell lines were validated by short tandem repeat DNA genotyping. All cells were cultured in Roswell Park Memorial Institute 1640 (Thermo Fisher Scientific, Waltham, MA, USA) supplemented with 10\% fetal bovine serum (FBS; Thermo Fisher Scientific), $100 \mathrm{IU} / \mathrm{mL}$ penicillin (Sigma, St Louis, MO, USA), and 100 $\mu \mathrm{g} / \mathrm{mL}$ streptomycin (Sigma). Details of cell culture were described previously. ${ }^{13}$

\section{Plasmid transfection and siRNA treatment}

The plasmids pCMV6-ddk-myc and pCMV6-ddk-mycFAM98A were obtained from Origene (RC209306; Origene, Rockville, MD, USA). FAM98A-siRNA (sc-94295) and negative control-siRNA (sc-37007) were obtained from Santa Cruz Biotechnology (Dallas, TX, USA). Transient transfection was performed using Lipofectamine 3000 (Thermo Fisher Scientific) according to the manufacturer's instructions. Cells were collected $48 \mathrm{~h}$ after plasmid transfection. For siRNA treatment, cells were collected $72 \mathrm{~h}$ after transfection.

\section{Immunohistochemistry}

Samples were fixed, embedded, and sliced into 4- $\mu \mathrm{m}$ thick tissue sections. Immunostaining was carried out using the streptavidin-peroxidase method. Monoclonal rabbit antiFAM98A antibody (ab204083; Abcam, Cambridge, MA, USA) was applied at a dilution of $1: 100$ in $1 \%$ bovine serum albumin (BSA)-PBS for incubation at $4^{\circ} \mathrm{C}$ overnight. 
Tissue sections were washed twice in PBS buffer, and secondary anti-rabbit IgG antibody conjugated to horseradish peroxidase was used for the following incubation (MaiXin, Fuzhou, China). Two investigators, who were blind to the clinical data, performed semi-quantitative scoring of the slides by evaluating the staining intensity and determining the percentage of stained cells in representative areas. Staining intensity was scored as 0 (no signal), 1 (weak), 2 (moderate), or 3 (high). The percentage of positively stained cells was scored as 0 (no signal), 1 (1\%-25\%), 2 $(26 \%-50 \%), 3(51 \%-75 \%)$, or $4(76 \%-100 \%)$. Final scores, which ranged from 0 to 12 , were obtained by multiplying the intensity and percentage scores. Tumors with scores $\geq 4$ were considered to exhibit positive FAM98A expression. Tumor samples with scores between 1 and 3 were considered to exhibit weak FAM98A expression, whereas those with scores of 0 were considered to have negative expression. For statistical analysis, FAM98A expression was divided into 2 groups (positive and negative expression). The positive group included tumors with scores $\geq 4$, and the negative group included tumors with scores between 0 and 3 . The details were described previously. ${ }^{13}$

\section{Western blotting}

Total protein was harvested, extracted, and quantified according to the manufacturer's instructions. ${ }^{14}$ Protein samples (80 $\mu \mathrm{g})$ were separated by $10 \%$ sodium dodecyl sulfate-polyacrylamide gel electrophoresis, and then transferred onto polyvinylidene fluoride membranes (Millipore, Billerica, MA, USA), then blocked with 5\% non-fat milk in tris-buffered saline with Tween 20. Membranes were incubated with primary antibodies to FAM98A (1:500, Abcam), phosphorylated P38 (p-P38), P38, phosphorylated ATF2 (p-ATF2), ATF2, cyclin D1 (1:1000; Cell Signaling Technology, Danvers, MA, USA), glyceraldehyde-3-phosphate dehydrogenase (1:2000, BD Transduction Laboratories, Lexington, KY, USA), and $\beta$-actin (1:500; Santa Cruz Biotechnology) overnight at $4^{\circ} \mathrm{C}$. The P38 inhibitor sb203580 was purchased from Cell Signaling Technology. Next, membranes were washed and incubated with peroxidase-conjugated anti-mouse or anti-rabbit IgG at $37^{\circ} \mathrm{C}$ for $2 \mathrm{~h}$ (1:2000, ZSGB-BIO, Beijing, China). Protein bands were quantified using a bio-imaging system (DNR Bio-Imaging Systems, Jerusalem, Israel).

\section{Immunofluorescence staining}

Cells were washed with PBS, fixed with $4 \%$ paraformaldehyde, and blocked with 5\% BSA for $1 \mathrm{~h}$. Afterward, the cells were incubated overnight with FAM98A antibodies (1:50) at $4^{\circ} \mathrm{C}$. Cells were washed with PBS and incubated with tetramethylrhodamine isothiocyanate-conjugated secondary antibodies (Cell Signaling Technology) at $37^{\circ} \mathrm{C}$ for $2 \mathrm{~h}$. Cell nuclei were counterstained with 4',6-diamidino-2-phenylindole. Epifluorescence microscopy was performed using an inverted Nikon TE300 microscope (Nikon Co., Ltd., Tokyo, Japan), and confocal microscopy was performed using a Radiance 2000 laser scanning confocal microscope (Carl Zeiss, Oberkochen, Germany).

\section{MTT assay}

Cells were plated in 96-well plates in media containing $10 \%$ FBS at $\sim 1 \times 10^{3}$ cells per well $24 \mathrm{~h}$ after transfection. Cells were cultured for $24 \mathrm{~h}$. MTT solution $(20 \mu \mathrm{L}, 5 \mathrm{mg} / \mathrm{mL})$ was added to every well and incubated for $4 \mathrm{~h}$ at $37^{\circ} \mathrm{C}$. Culture media were removed from each well, and the resulting MTT formazan product was solubilized in $150 \mu \mathrm{L}$ of dimethyl sulfoxide. The results were quantitated spectrophotometrically at a wavelength of $490 \mathrm{~nm}$.

\section{Colony formation assay}

A549 and SPC cells were transfected with pCMV6 or pCMV6-FAM98A plasmids, a negative control, or FAM98AsiRNA for $48 \mathrm{~h}$. Cells were plated in 6-cm cell culture dishes at 1000 cells per dish. Cells were incubated for 12 days. The plates were washed with PBS and stained with Giemsa. The number of colonies containing $>50$ cells was counted under the microscope manually.

\section{Statistical analysis}

The association between FAM98A and clinicopathologic features was measured by Pearson's chi-square test. The imaging results of Western blotting and the results of invasion assay were analyzed using the Mann-Whitney $U$-test. A $p$-value $<0.05$ indicated statistical significance. All statistical analyses were performed using SPSS version 17.0 for windows (IBM Corporation, Armonk, NY, USA).

\section{Results FAM98A was overexpressed in NSCLC specimens and the A549 and SPC-AI cell lines}

FAM98A protein levels were measured in 20 fresh NSCLC specimens and corresponding noncancerous lung tissues using Western blotting (Figure 1A). In 15 specimens, higher FAM98A protein expression was detected in NSCLC tissues than the adjacent noncancerous lung tissues $(p<0.05$; Figure 1B). 
Immunohistochemical analysis was utilized to determine FAM98A expression level and subcellular localization in 128 NSCLC and corresponding noncancerous lung tissues. Negative or weak expression of FAM98A was detected in normal alveolar epithelial and bronchial ciliated columnar epithelial cells, located in the cytoplasm. FAM98A expression was higher in lung carcinoma and adenocarcinoma tissues than the adjacent noncancerous tissues, also in the cytoplasm (Figure 1C). Additionally, we investigated FAM98A expression in 7 NSCLC cell lines (the lung adenocarcinoma cell lines A549, H1299, 973, 83A, and SPC-A1, the lung large cell carcinoma cell line $\mathrm{H} 460$, the lung squamous cell carcinoma cell line LK2)

A

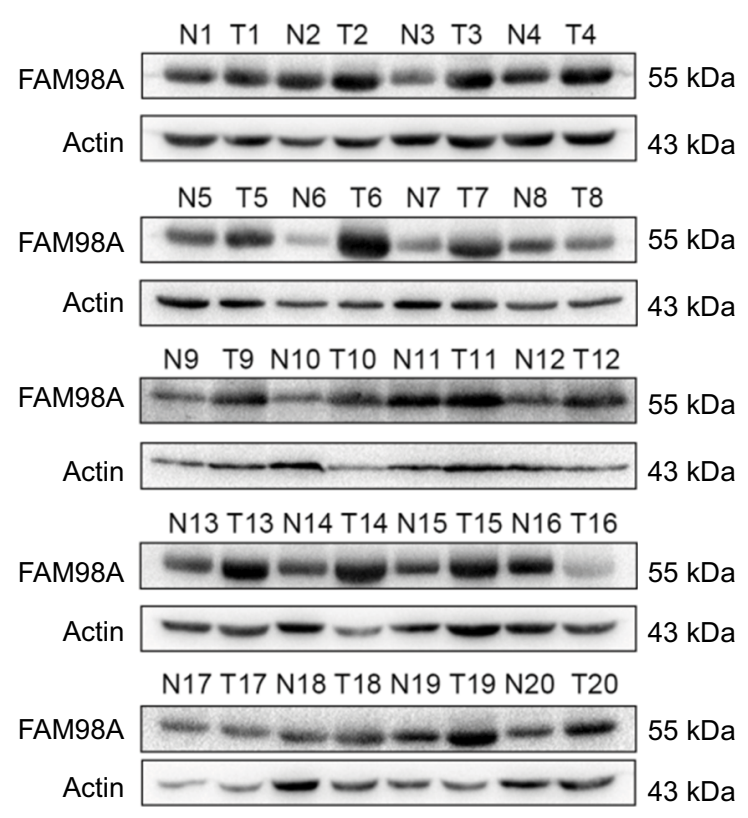

C

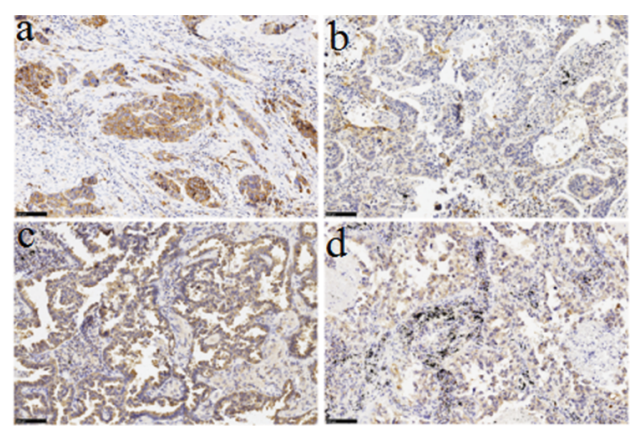

and the HBE line of healthy cells. The expression level of FAM98A was higher in A549, SPC-A1, 973, 83A, and LK2 cell lines than the HBE cell line, and it was primarily located in the cytoplasm (Figure 1D, E). However, the expression of FAM98A was lower in both the H1299 and H460 cell lines (Figure 1D).

Statistical analysis of the clinicopathologic data indicated that positive FAM98A expression in the cytoplasm was significantly related to tumor TNM stage $(p=0.017)$ and lymph node metastasis $(p<0.001)$. However, there was no association between its expression and patient age, gender, histologic type, or differentiation ( $p>0.05$; Table 1). Expression of FAM98A was significantly higher in NSCLC

B
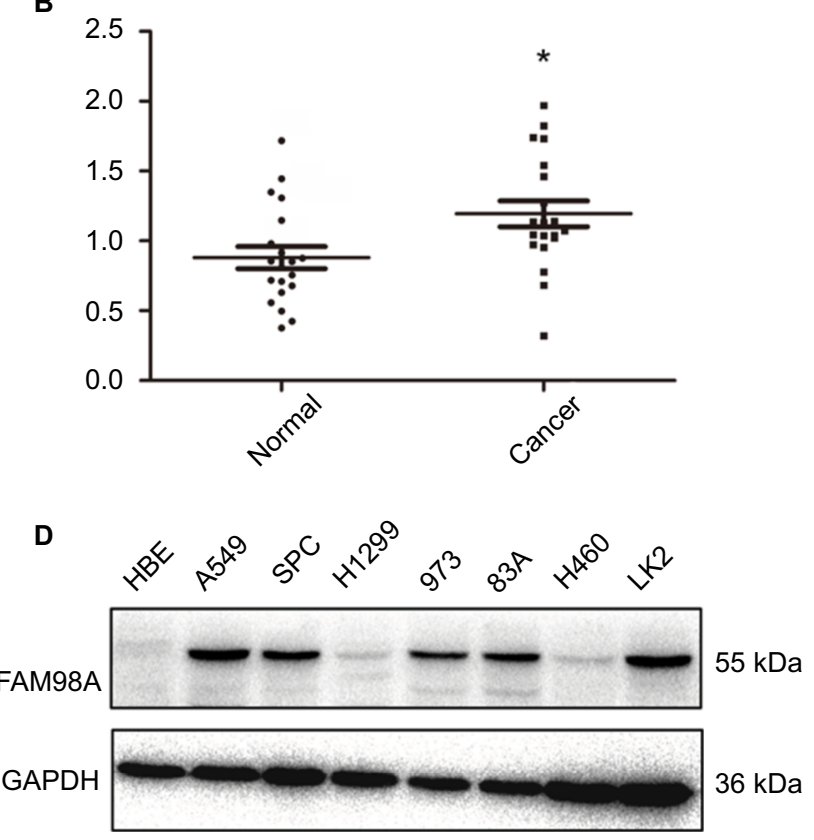

E

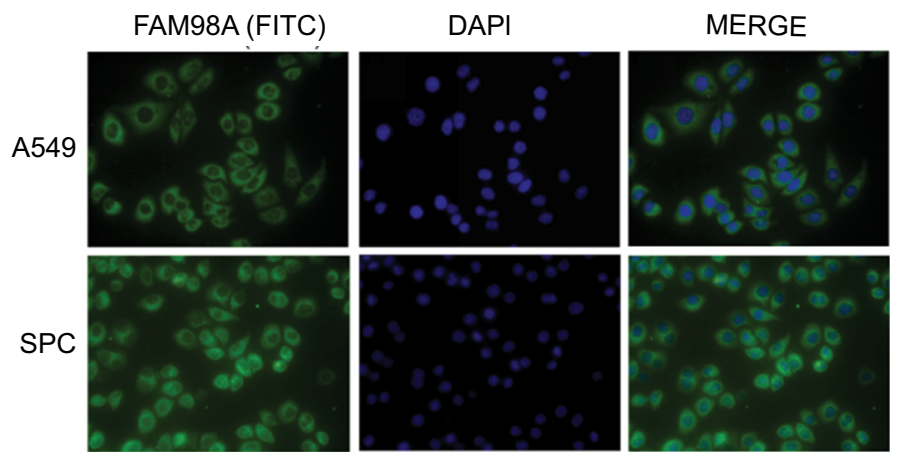

Figure I FAM98A expression in NSCLC specimens and cell lines.

Notes: (A) and (B) Protein expression of FAM98A in NSCLC tissues was considerably higher than in noncancerous tissues. *Mean $p<0.05$. (C) Results showed low FAM98A expression in (a) normal bronchial epithelial cells and (b) normal alveolar epithelial. However, FAM98A was highly expressed in (c) lung squamous cell carcinoma and (d) adenocarcinoma. (D) FAM98A was expressed in different cell lines. (E) FAM98A was localized in the cytoplasm of A549 and SPC cells.

Abbreviations: DAPI, 4',6-diamidino-2-phenylindole; GAPDH, glyceraldehyde-3-phosphate dehydrogenase; FITC, fluorescein isothiocyanate; NSCLC, non-small cell lung cancer; HBE, human bronchial epithelial. 
samples than the noncancerous samples $(118 / 128$ vs $10 / 128$; $p<0.001$; Table 2).

\section{Overexpression of FAM98A enhanced proliferation and colony formation of NSCLC cell lines}

We explored the role of FAM98A in the proliferation and colony formation abilities of A549 and SPC-A1 cells after transfection with a FAM98A overexpression plasmid or FAM98A siRNA (Figure 2A). The MTT assay and colony formation assay results showed that overexpressing FAM98A promoted the proliferation and colony formation abilities of both A549 and SPC-A1 cells, whereas downregulation of FAM98A inhibited their proliferation and colony formation abilities (Figure 2B, C).

Table I Correlation of FAM98A expression with clinicopathologic features in $13 \mathrm{I}$ cases of lung cancer

\begin{tabular}{lllll}
\hline Factors & N & FAM98A & \\
\cline { 2 - 5 } & & Negative & Positive & p-value \\
\hline Total & I3I & 45 & 86 & \\
Age (years) & & & & 0.215 \\
$\quad \leq 60$ & 68 & 26 & 42 & \\
$>60$ & 63 & 19 & 44 & \\
Gender & & & & 0.576 \\
$\quad$ Male & 102 & 35 & 67 & \\
$\quad$ Female & 29 & 10 & 19 & \\
Histological type & & & & 0.714 \\
$\quad$ Squamous cell & 60 & 18 & 42 & \\
carcinoma & & & & \\
$\quad$ Adenocarcinoma & 62 & 23 & 39 & \\
$\quad$ Others & 9 & 4 & 5 & \\
Differentiation & & & & 0.654 \\
$\quad$ Well & 15 & 5 & 10 & \\
$\quad$ Moderate & 59 & 18 & 41 & \\
$\quad$ Poor & 57 & 22 & 35 & \\
TNM stage & & & & 0.017 \\
$\quad$ I and II & 90 & 65 & 25 & \\
III & 41 & 20 & 21 & \\
Lymph node metastasis & & & & \\
$\quad$ No & 73 & 36 & 37 & \\
$\quad$ Yes & 58 & 9 & 49 & \\
\hline
\end{tabular}

Table 2 The comparison of FAM98A levels in the NSCLC and corresponding adjacent tissues

\begin{tabular}{llllll}
\hline Group & N & FAM98A & & \multirow{2}{*}{$\chi^{2}$} & p-value \\
\cline { 2 - 4 } & & Negative & Positive & & \\
\hline NSCLC & 128 & 20 & 108 & 106.61 & $<0.00 I^{\mathrm{a}}$ \\
Adjacent tissues 128 & I I8 & 10 & & \\
\hline
\end{tabular}

Note: a Mean that $p<0.05$ was statistically significant.

Abbreviation: NSCLC, non-small cell lung cancer.

\section{FAM98A enhanced cyclin DI via} activating the P38-ATF2 pathway

Finally, the key signaling pathway factors and relevant cell cycle proteins were screened by transfecting A549 and SPC-A1 cells with the FAM98A overexpression plasmid or FAM98A siRNA (Supplementary material). Overexpression of FAM98A increased p-P38 and p-ATF2 expression, and cyclin D1. Correspondingly, depleting FAM98A decreased p-P38, p-ATF2 expression, and cyclin D1 (Figure 3A).

We added the P38-specific inhibitor sb203580 to the culture medium after FAM98A overexpression in A549 and SPC-A1 cells to validate whether the upregulation of cyclin D1 induced by FAM98A was owing to activation of the P38 signaling pathway. The $\mathrm{p} 38$ inhibitor significantly prevented the phosphorylation of ATF2 and cyclin D1 (Figure 3B). Tumor proliferation induced by FAM98A overexpression was, in turn, reversed by treatment with the P38 inhibitor (Figure 3C).

\section{Discussion}

Overexpression of FAM98A was detected in the cytoplasm of NSCLC tissues and correlated to advanced TNM staging and positive lymph node metastasis. These observations suggested that FAM98A overexpression may play a role in the occurrence of NSCLC as a precursor. FAM98A facilitated cyclin D1 expression via activating the P38-ATF2 signaling pathway. Cyclin D1 is a key protein in the G1/S checkpoint and promotes cell cycle progression from $\mathrm{G} 1$ to $\mathrm{S}$ phase. ${ }^{15,16}$ Thus, it appears that FAM98A could potentially play a role in promoting the etiology of NSCLC by upregulating the expression of cyclin D1.

A previous study showed that FAM98A is expressed in ovarian cancer cell lines and is important for migration, invasion, and colony formation characteristics. ${ }^{6}$ Moreover, FAM98A is expressed in colorectal cancer tissues, and its knockdown suppresses proliferation and colony formation in both HCT116 and HT29 cells by reducing PRMT1 expression and arginine methylation. ${ }^{17}$ The aforementioned reports were consistent with our observation that FAM98A was expressed in tumor tissues and was a factor in promoting tumor development. Unfortunately, its precise physiological functions remain unknown and more effort is needed to investigate its characteristics in vivo and in vitro. FAM98A and its homolog FAM98B are binding partners of PRMT1 and share $58 \%$ amino acid sequence identity. ${ }^{17}$ FAM98B forms a complex with DDX1, HSPC117, and C14orf166 shuttles, which play a dominant role in the fate of nuclear and 
cytoplasmic RNA, ${ }^{18}$ and knockdown suppresses accumulation of DDX1. ${ }^{19}$ Thus, we inferred that FAM98A performs a role similar to FAM98B, considering that they contain $58 \%$ identical amino acid sequences.

Overexpression of FAM98A increased p-P38 and $\mathrm{p}-\mathrm{ATF} 2$, which is a downstream factor of $\mathrm{p}-\mathrm{P} 38$. Furthermore, treatment with a P38-specific inhibitor after transfection with the FAM98A plasmid reversed the upregulation of cyclin D1 and the proliferating and colony formation abilities of A549 and SPC-A1 cells. Additionally, increasing cyclin D1 promotes cell proliferation through involvement of the $\mathrm{PI} 3 \mathrm{~K} / \mathrm{AKT}$ signaling pathway. ${ }^{20}$ These data were consistent with previously published reports that the P38-ATF2 signaling pathway promotes proliferation via transcriptional activation of key cell-cycle regulators. ${ }^{21-23}$ A previous report showed that FAM98A, as a novel substrate of PRMT1, is arginine-methylated by PRMT1. ${ }^{6}$ PRMT1 is associated with p38 $\alpha$ in cells, as shown by co-immunoprecipitation, and directly methylates $\mathrm{p} 38 \alpha$ in in vitro methylation assays. ${ }^{24}$ Moreover, the activation of PRMT1 and PRMT5 mediates hypoxia- and ischemia-induced apoptosis in human lung epithelial cells and the lung tissue of miniature pigs via p-p38 mitogen-activated protein kinase (MAPK) and $\mathrm{p}-\mathrm{JNK} .{ }^{25}$ In addition, PRMT1 suppresses megakaryocytic differentiation
A

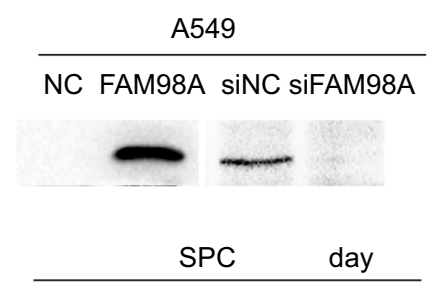

NC FAM98A SiNC SiFAM98A

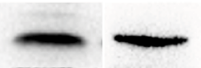

B

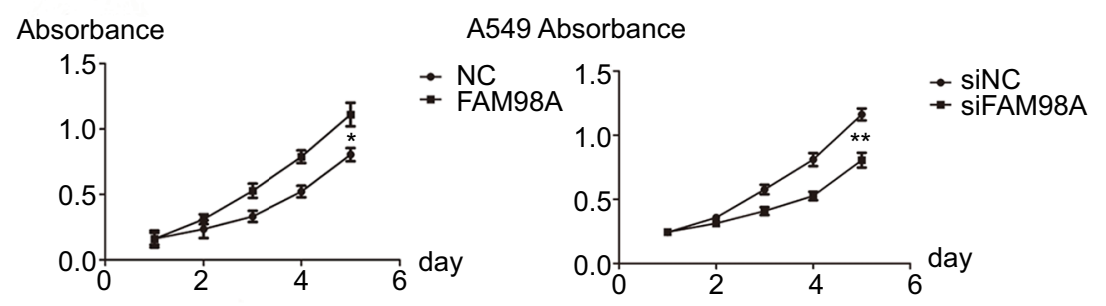

SPC

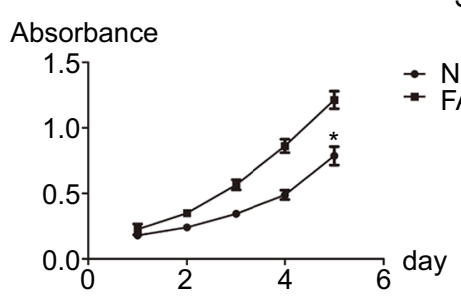

Absorbance

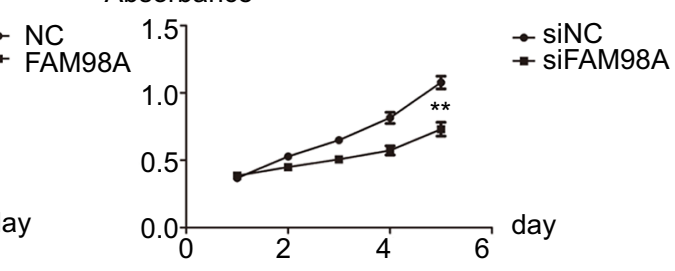

C
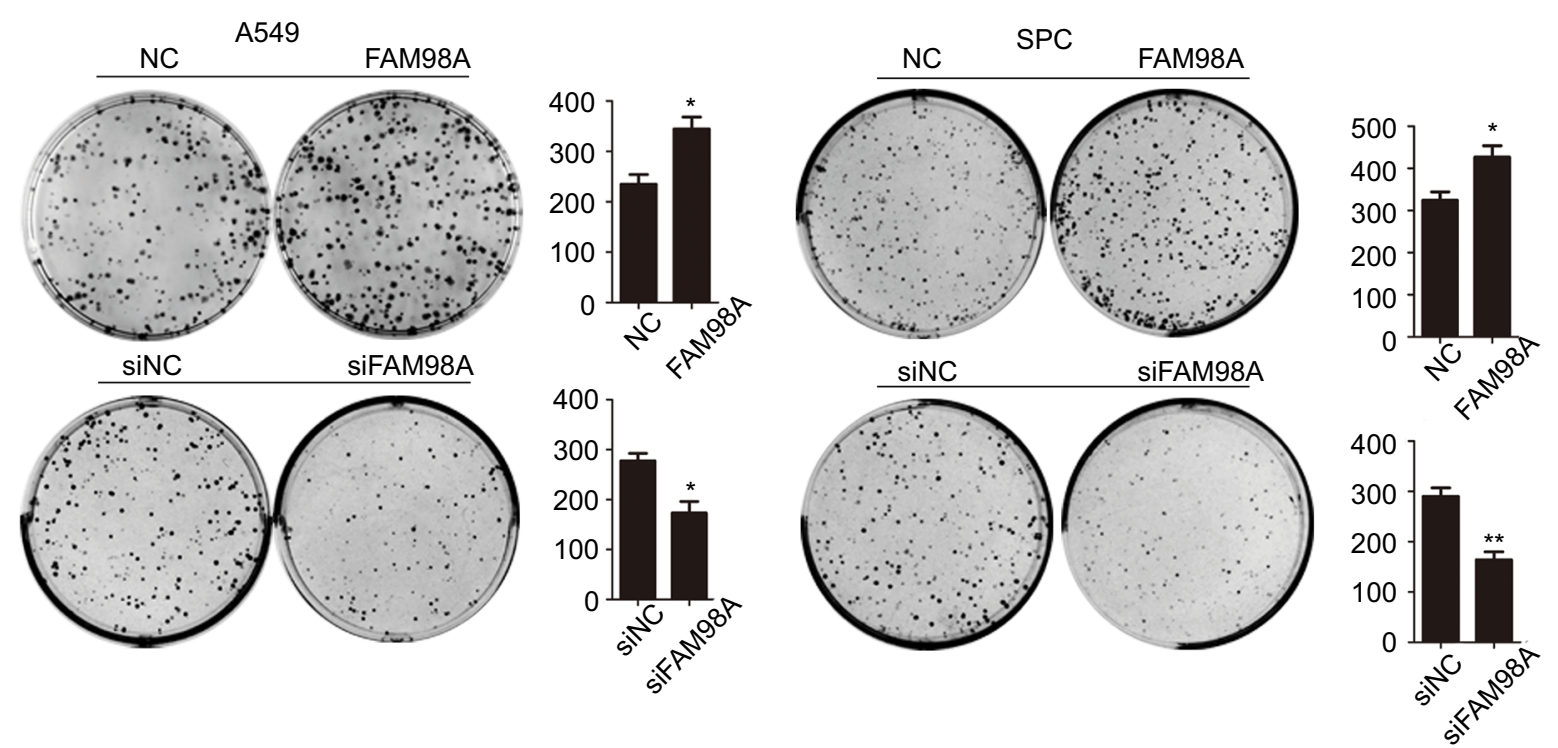

Figure 2 FAM98A promoted the proliferation of A549 and SPC cells.

Notes: (A) Transfection efficiencies after FAM98A overexpression and depletion by siRNA treatment in A549 and SPC cells were determined by Western blotting. (B) and (C) The proliferation and colony-formation abilities of A549 and SPC cells increased after overexpressing FAM98A and decreased when treated with siRNA targeting FAM98A. The assays were replicated 3 times. *Mean $p<0.05 ; * *$ mean $p<0.01$.

Abbreviation: NC, negative control. 
A
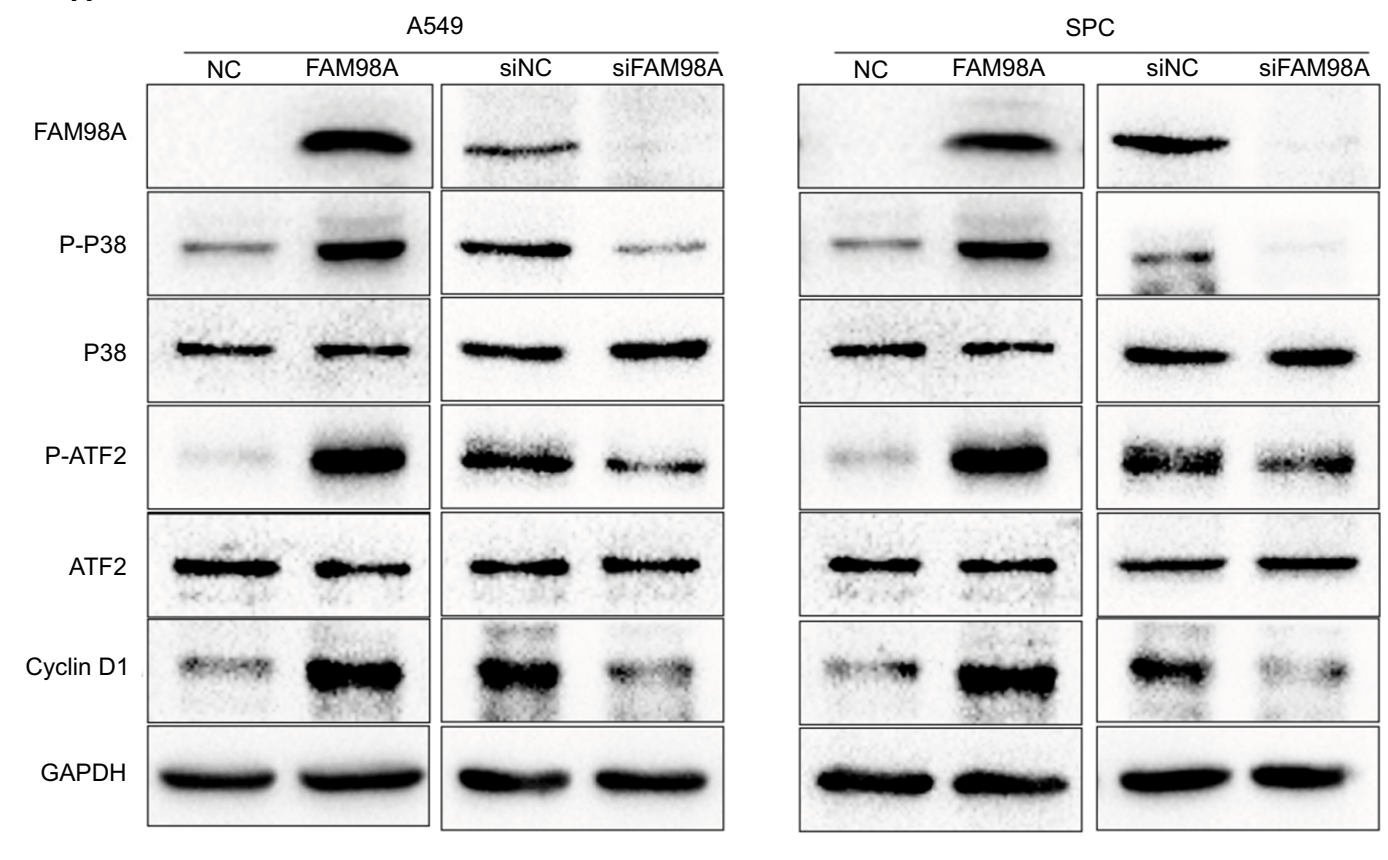

B
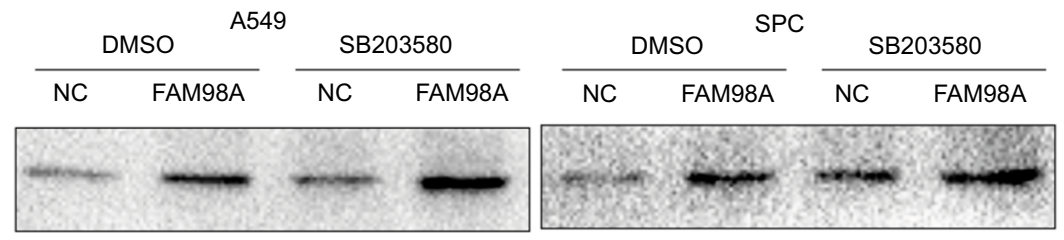

P-ATF2
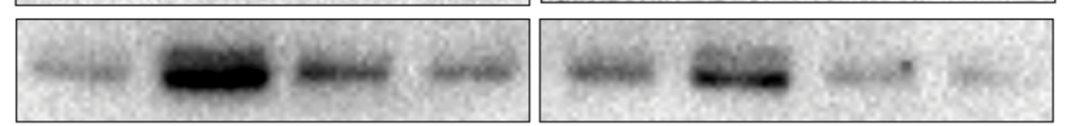

Cyclin D1
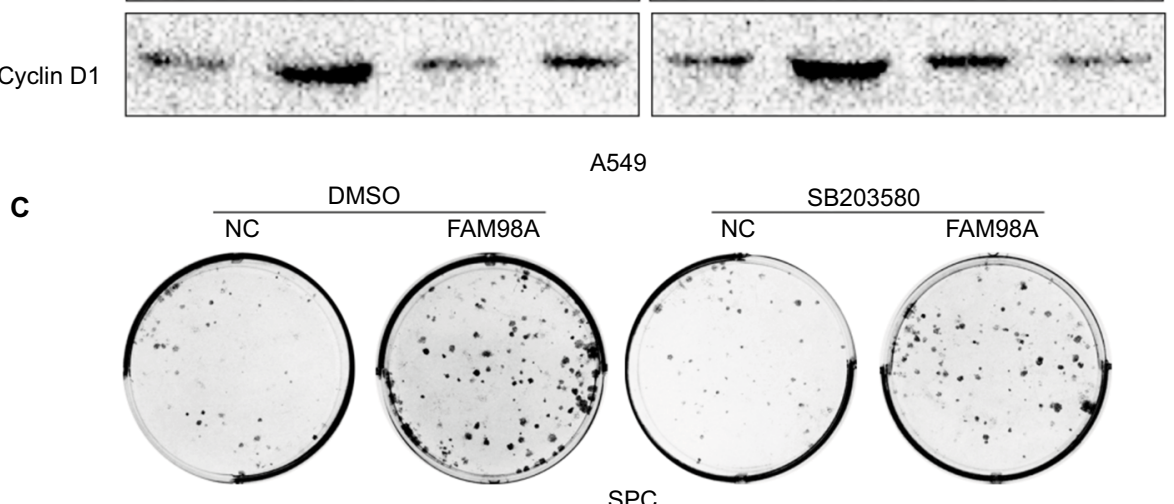

A549

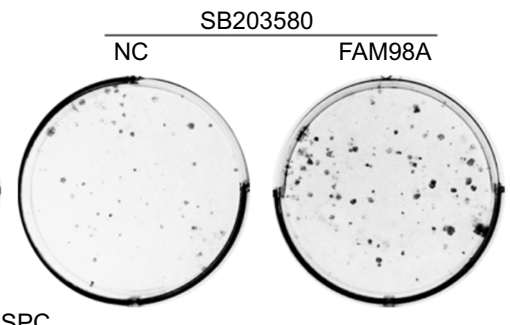

SPC

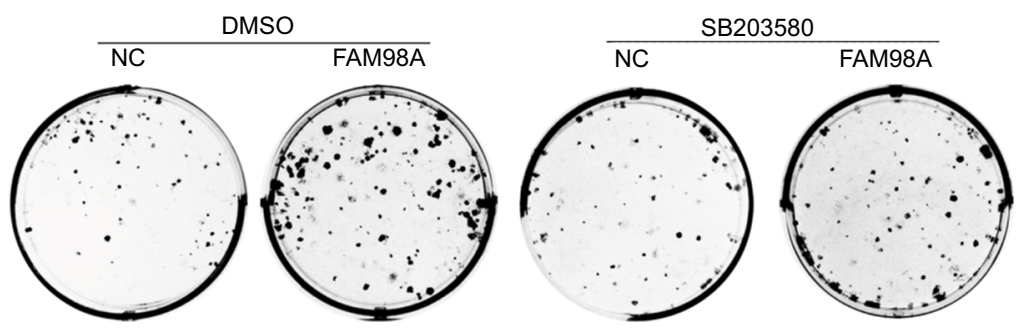

Figure 3 FAM98A increased cyclin DI expression and enhanced proliferation via promoting P38-ATF2 phosphorylation.

Notes: (A) The expression of p-P38, p-ATF2, and cyclin DI were determined after FAM98A overexpression or knockdown in A549 and SPC cells. (B) The expressions of p-P38, p-ATF2, and cyclin DI were measured after SB203580, a P38 inhibitor, was added to the culture media of A549 and SPC cells with or without FAM98A overexpression. DMSO was used as negative control. (C) The colony formation assay was performed after SB203580, a P38 inhibitor, was added to the culture media of A549 and SPC cells with or without FAM98A overexpression. DMSO was used as negative control. The assays were replicated 3 times.

Abbreviations: DMSO, dimethyl sulfoxide; GAPDH, glyceraldehyde-3-phosphate dehydrogenase; p-ATF2, phosphorylated ATF2; p-P38, phosphorylated P38; NC, negative control. 
via modulation of the p38 MAPK pathway in K562 cells. ${ }^{26}$ Therefore, we inferred that FAM98A could increase the expression of cyclin D1 by activating the P38-ATF2 signaling pathway and subsequently enhancing tumor cell proliferation. These conclusions need to be validated by additional functional experiments in future.

\section{Conclusion}

Our results showed that FAM98A is overexpressed in the cytoplasm of NSCLC tissues and correlated with advanced TNM staging and positive lymph node metastasis. Thus, FAM98A may increase the expression of cyclin D1 by activating the P38-ATF2 signaling pathway and subsequently enhancing tumor cell proliferation; these results require further validation.

\section{Acknowledgment}

This study was supported by Natural Science Foundation of Liaoning Province of China (No. 2014021013).

\section{Disclosure}

The authors report no conflicts of interest in this work.

\section{References}

1. Jemal A, Bray F, Center MM, Ferlay J, Ward E, Forman D. Global cancer statistics. CA Cancer J Clin. 2011;61(2):69-90.

2. Sangodkar J, Katz S, Melville H, Narla G. Lung adenocarcinoma: lessons in translation from bench to bedside. Mt Sinai J Med. 2010;77(6): 597-605.

3. Schou KB, Andersen JS, Pedersen LB. A divergent calponin homology (NN-CH) domain defines a novel family: implications for evolution of ciliary IFT complex B proteins. Bioinformatics. 2014;30(7):899-902.

4. Chen M, Ye Y, Zou B, et al. C14orf166 is a high-risk biomarker for bladder cancer and promotes bladder cancer cell proliferation. J Transl Med. 2016;14:55.

5. Fujiwara T, Ye S, Castro-Gomes T, et al. PLEKHM1/DEF8/RAB7 complex regulates lysosome positioning and bone homeostasis. JCI Insight. 2016;1(17):e86330.

6. Akter KA, Mansour MA, Hyodo T, Ito S, Hamaguchi M, Senga T. FAM98A is a novel substrate of PRMT1 required for tumor cell migration, invasion, and colony formation. Tumour Biol. 2016;37(4): 4531-4539.

7. Cheeseman IM, Desai A. Molecular architecture of the kinetochoremicrotubule interface. Nat Rev Mol Cell Biol. 2008;9(1):33-46.

8. Pesenti ME, Weir JR, Musacchio A. Progress in the structural and functional characterization of kinetochores. Curr Opin Struct Biol. 2016;37:152-163.
9. Cheeseman IM. The kinetochore. Cold Spring Harb Perspect Biol. 2014;6(7):a015826.

10. Martin-Lluesma S, Stucke VM, Nigg EA. Role of Hec1 in spindle checkpoint signaling and kinetochore recruitment of Mad1/Mad2. Science. 2002;297(5590):2267-2270.

11. Travis WD, Brambilla E, Burke AP, Marx A, Nicholson AG. Introduction to the 2015 world health organization classification of tumors of the lung, pleura, thymus, and heart. JThorac Oncol. 2015;10(9):1240-1242.

12. Goldstraw P. Updated staging system for lung cancer. Surg Oncol Clin NAm. 2011;20(4):655-666.

13. Shan Y, Cao W, Wang T, Jiang G, Zhang Y, Yang X. ZNF259 inhibits non-small cell lung cancer cells proliferation and invasion by FAK-AKT signaling. Cancer Manag Res. 2017;9:879-889.

14. Bradford MM. A rapid and sensitive method for the quantitation of microgram quantities of protein utilizing the principle of protein-dye binding. Anal Biochem. 1976;72:248-254.

15. Chai XM, Li YL, Chen H, Guo SL, Shui LL, Chen YJ. Cigarette smoke extract alters the cell cycle via the phospholipid transfer protein/ transforming growth factor-beta1/CyclinD1/CDK4 pathway. Eur $J$ Pharmacol. 2016;786:85-93.

16. Wu W, Liu Q, Liu Y, Yu Z, Wang Y. Dixdc1 targets CyclinD1 and $\mathrm{p} 21$ via PI3K pathway activation to promote Schwann cell proliferation after sciatic nerve crush. Biochem Biophys Res Commun. 2016;478(2):956-963.

17. Akter KA, Mansour MA, Hyodo T, Senga T. FAM98A associates with DDX1-C14orf166-FAM98B in a novel complex involved in colorectal cancer progression. Int J Biochem Cell Biol. 2017;84:1-13.

18. Perez-Gonzalez A, Pazo A, Navajas R, Ciordia S, Rodriguez-Frandsen A, Nieto A. hCLE/C14orf166 associates with DDX1-HSPC117-FAM98B in a novel transcription-dependent shuttling RNA-transporting complex. PLoS One. 2014;9(3):e90957.

19. Popow J, Englert M, Weitzer S, et al. HSPC117 is the essential subunit of a human tRNA splicing ligase complex. Science. 2011;331(6018):760-764.

20. Zhang W, Lei C, Fan J, Wang J. miR-18a promotes cell proliferation of esophageal squamous cell carcinoma cells by increasing cylin D1 via regulating PTEN-PI3K-AKT-mTOR signaling axis. Biochem Biophys Res Commun. 2016;477(1):144-149.

21. Hsu MK, Qiao L, Ho V, et al. Ethanol reduces p38 kinase activation and cyclin D1 protein expression after partial hepatectomy in rats. $J$ Hepatol. 2006;44(2):375-382.

22. Recio JA, Merlino G. Hepatocyte growth factor/scatter factor activates proliferation in melanoma cells through $\mathrm{p} 38 \mathrm{MAPK}, \mathrm{ATF}-2$ and cyclin D1. Oncogene. 2002;21(7):1000-1008.

23. Zhang X, Zhang Y, Fan C, et al. Noxin promotes proliferation of breast cancer cells via P38-ATF2 signaling pathway. Tumour Biol. 2017;39(6): 1010428317705515.

24. Hua WK, Chang YI, Yao CL, Hwang SM, Chang CY, Lin WJ. Protein arginine methyltransferase 1 interacts with and activates p38alpha to facilitate erythroid differentiation. PLoS One. 2013;8(3): e56715.

25. Lim SK, Jeong YW, Kim DI, et al. Activation of PRMT1 and PRMT5 mediates hypoxia- and ischemia-induced apoptosis in human lung epithelial cells and the lung of miniature pigs: the role of $\mathrm{p} 38$ and JNK mitogen-activated protein kinases. Biochem Biophys Res Commun. 2013;440(4):707-713.

26. Chang YI, Hua WK, Yao CL, et al. Protein-arginine methyltransferase 1 suppresses megakaryocytic differentiation via modulation of the $\mathrm{p} 38$ MAPK pathway in K562 cells. J Biol Chem. 2010;285(27):20595-20606. 


\section{Supplementary material}

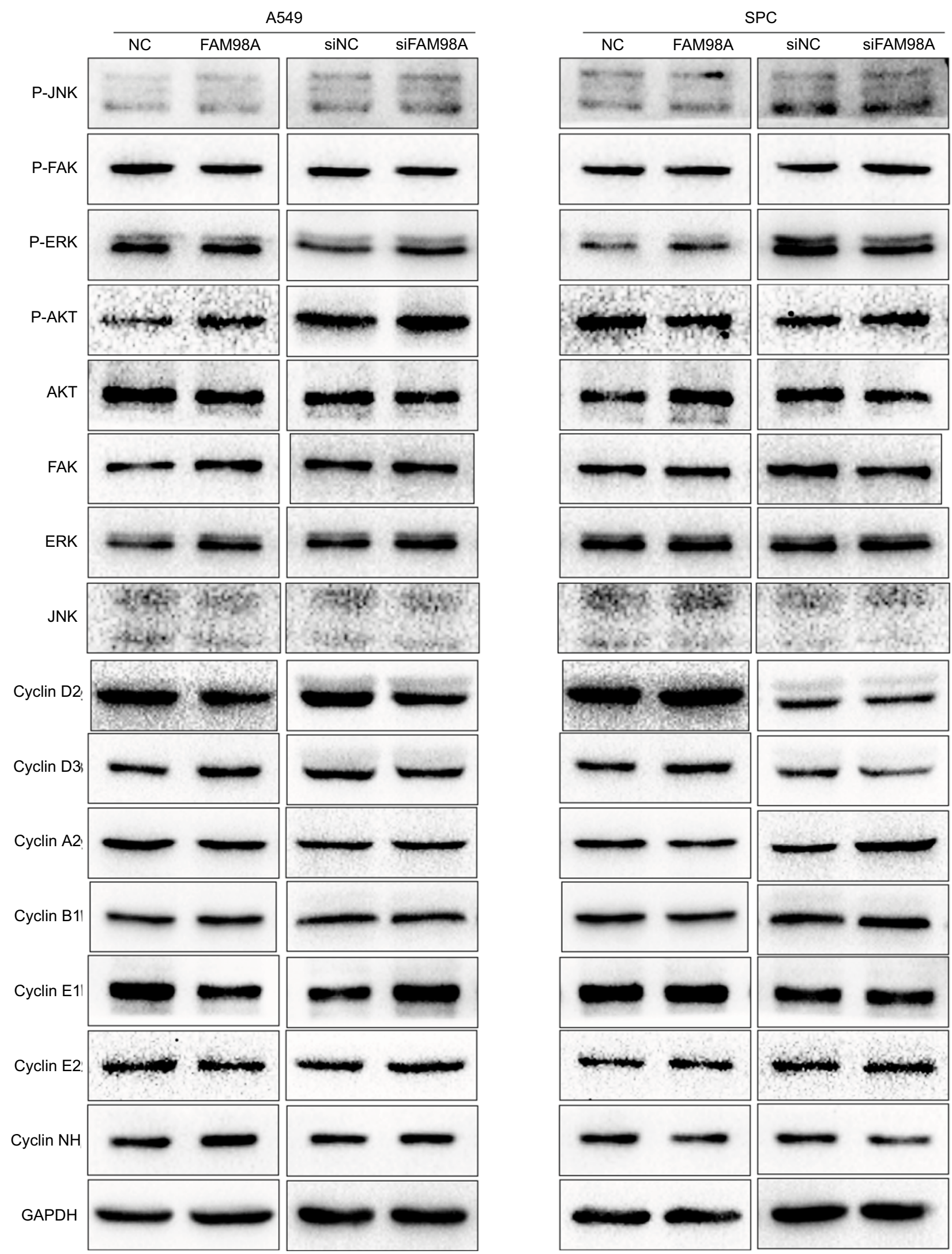

Figure SI The protein expressions related to signal pathway and cell cycle after FAM98A overexpression and depletion by siRNA treatment in A549 and SPC cells. Abbreviations: FAK, focal adhesion kinase; ERK, extracellular signal-regulated kinase; GAPDH, glyceraldehyde-3-phosphate dehydrogenase; JNK, c-Jun N-terminal kinase; NC, negative control. 


\section{Publish your work in this journal}

Cancer Management and Research is an international, peer-reviewed open access journal focusing on cancer research and the optimal use of preventative and integrated treatment interventions to achieve improved outcomes, enhanced survival and quality of life for the cancer patient. The manuscript management system is completely online and includes a very quick and fair peer-review system, which is all easy to use. Visit http://www.dovepress.com/testimonials.php to read real quotes from published authors. 